\title{
Utilizing nonlinear optical microscopy to investigate the development of early cancer in nude mice in vivo
}

\author{
Chun-Chin Wang*a, Feng-Chieh Li ${ }^{\mathrm{a}}$, Sung-Jan Lin ${ }^{\mathrm{b}}$, Wen Lo ${ }^{\mathrm{a}}$, Chen-Yuan Dong ${ }^{\mathrm{a}}$ \\ ${ }^{a}$ Department of Physics, National Taiwan University, Taipei, Taiwan, R.O.C.; \\ ${ }^{b}$ Department of Dermatology, National Taiwan University Hospital and College of Medicine, \\ Taipei, Taiwan, R.O.C.
}

\begin{abstract}
In this investigation, we used in vivo nonlinear optical microscopy to image normal and carcinogen DMBA treated skin tissues of nude mice. We acquired two-photon autofluroescence and second harmonic generation (SHG) images of the skin tissue, and applied the ASI (Autofluorescence versus SHG Index) to the resulting image. This allows us to visualize and quantify the interaction between mouse skin cells and the surrounding connective tissue.

We found that as the imaging depth increases, ASI has a different distribution in the normal and the treated skin tissues. Since the DMBA treated skin eventually became squamous cell carcinoma (SCC), our results show that the physiological changes to mouse skin en route to become cancer can be effectively tracked by multiphoton microscopy. We envision this approach to be effective in studying tumor biology and tumor treatment procedures.
\end{abstract}

Keywords: Two-photon, second harmonic generation, skin, cancer, microscopy

\section{INTRODUCTION}

The golden standard for cancer diagnosis is through histological examination of biopsy specimens. However, histological biopsy is both invasive and time-consuming. To investigate the development of cancer or to find an early diagnosis of cancer, it is critical to obtain qualitative and quantitative information from the biological systems using intravital techniques.

Nonlinear optical microscopy such as two-photon fluorescence imaging has been used for biological specimen imaging both in vivo and in vitro. ${ }^{1,2}$ Due to the nonlinear mechanism, only the focal volume is effectively excited, resulting in optical sectioning capability with lower specimen photodamage.

In addition, noncentrosymmetric biological structures, such as collagen and muscle fibers are effective generators of second harmonic generation (SHG) signals. SHG is a non-invasive coherent process that is sensitive the structures of collagen fibers. Together with autofluorescence imaging, these two techniques form an effective and minimally invasive tool that is suitable for in vivo imaging, without the need of labeling dyes or pretreatment of samples.

In this study, we performed in vivo two-photon autofluorescence and SHG imaging of the dorsal skin of nude mice. With the use of a skinfold chamber, this method allows long term observation and can potentially be applied to reveal the mechanistic pathways of important physiological processes such as tumor metastasis.

Confocal, Multiphoton, and Nonlinear Microscopic Imaging III, edited by Tony Wilson, Ammasi Periasamy Proc. of SPIE-OSA Biomedical Optics, SPIE Vol. 6630, 66300Y, @ 2007 SPIE-OSA - 1605-7422/07/\$18 


\section{MATERIAL AND METHOD}

\section{Chamber Design}

Our version of the chamber design involves an observation window using a No. $1.5,15 \mathrm{~mm}$ circular cover glass. A cylinder-shape push piece located on the opposite side of chamber is used to adhere the tissue to the observation cover glass. To be compatible with biological samples, this chamber is made of medical-graded titanium alloy (6Al-4V, ELI). Our design is shown in Fig. 1.

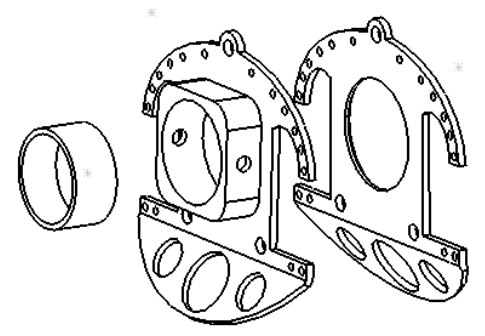

Fig.1. Design of dorsal skinfold chamber

\section{Animal Model}

Our design is intended to be used on 8-week-old nude mice (BALB/c-nu, National Animal Center, Taiwan). Preparations of these mice for imaging were all done in a laminar flow. Anesthesia was applied prior to mounting the dorsal skinfold chamber on the mouse. A stock solution of pentobarbital in phosphate buffered saline (PBS) at the concentration of $20 \mathrm{mg} / \mathrm{ml}$ was prepared. $80 \mu \mathrm{l}$ of the stock anesthesia solution was injected intraperitoneally.

After the application of the anesthesia the mouse was wiped with aqueous ethanol. To install the chamber, the rear mounting piece was first sutured onto the mouse. After a few days, the front mounting piece was also sutured onto the skin followed by the attachment of the mounting screws. Prior to installation, all chamber components were immersed in aqueous ethanol to minimize possible infections. After both the mounting pieces were attached, the skin exposed at the front mounting piece was partially removed. Finally, a No. 1.5 cover glass is mounted and secured with nail polish as shown in Fig. 2. ${ }^{3}$

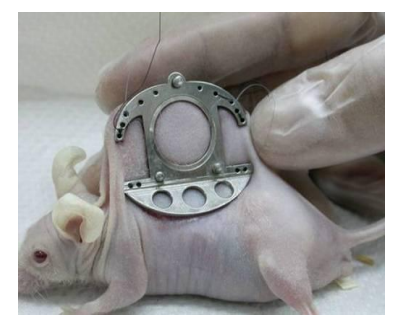

Fig.2. nude mouse mounted with dorsal skinfold chamber

\section{Experiment Setup}

The imaging system used in this study is a home-built laser scanning multiphoton microscope based on an upright microscope (E800, Nikon, Japan). The excitation source is a diode-pumped (Millennia X, Spectra Physics, Mountain View, California) Ti-sapphire laser (Tsunami, Spectra Physics). A schematic diagram of the multiphoton microscopic 
system is shown in Fig. 3. A laser beam with central wavelength of $780 \mathrm{~nm}$ is scanned in the focal plane by a galvanometer-driver x-y mirror scanning system (Model 6220, Cambridge Technology, Cambridge, Massachusetts). For high resolution imaging, a high-numerical-aperture, oil immersion objective (S Fluor 40, N.A. 1.3, Nikon) was used. The average power at the sample was about $10 \mathrm{~mW}$.

The generated autofluorescence (AF) and SHG signals are collected by the same objective. Before reaching the photomultiplier tube (PMT), the AF and SHG signals are separated by a dichroic mirror (435DCXR, Chroma Technology). The $390 \mathrm{~nm}$ SHG signal is reflected by the dichroic mirror and further filtered by a bandpass filter (HQ390/20, Chroma Technology), whereas AF signals pass through the dichroic mirror and is further filtered by a broad bandpass filter (E700sp-2P-E435lp, Chroma Technology). The signal photons are collected by a single-photon counting PMT (R7400P, Hamamatsu, Japan) and further processed by a discriminator before sending to the computer for image formation.

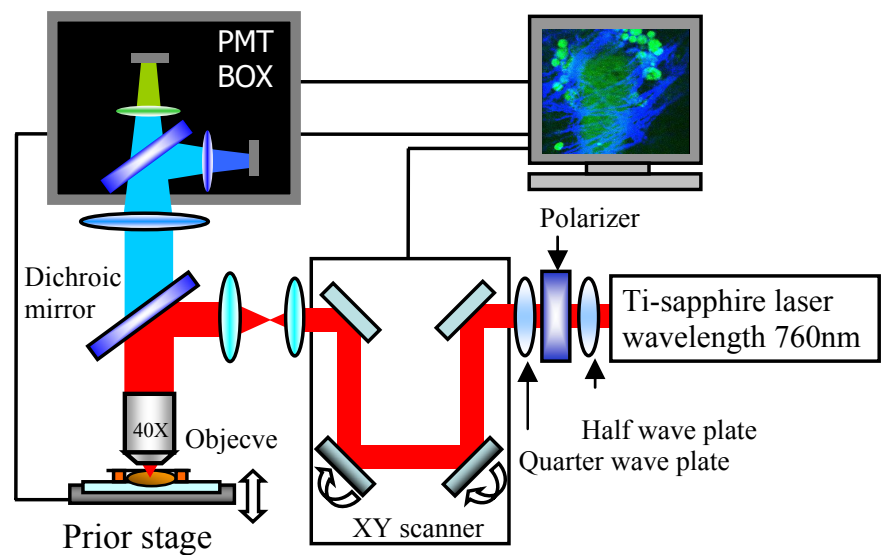

Fig.3. Multiphoton scanning system

\section{Cancer induction}

According to the paper published in Melanoma Research, Kligman reported the development of a malignant melanoma, with metastases, in a hairless mouse after six applications of $0.25 \%$ dimethylbenz [a] anthracene ( DMBA ) in acetone. ${ }^{4}$ In that study, all 12 DMBA-treated mice developed macules, papules and metastatic melanoma, whereas the acetonetreated controls developed no lesions. Due to the success of this method for inducing cancer, we choose it as the protocol for this experiment.

\section{RESULTS}

After five weeks and six applications of DMBA, we mounted a dorsal skinfold chamber onto the mouse. We obtained 3D images of normal and DMBA treated skin by the use of nonlinear optical microscopy. Combined two-photon fluorescence with SHG signals, we can simultaneously obtain molecular and structural information of the specimen. The images shown in Fig.4 and Fig.5 are the three dimensional images from stratum corneum to the dermis. The average power at the sample is about $10 \mathrm{~mW}$. We obtained a series of autofluorescence (from $435 \mathrm{~nm}$ to $700 \mathrm{~nm}$ to acquire most intrinsic fluorescence) and SHG images. The in vivo observation indicated that SHG signal (blue pseudocolor) in the dermis of normal nude mice is quite different than that of DMBA treated skin. 

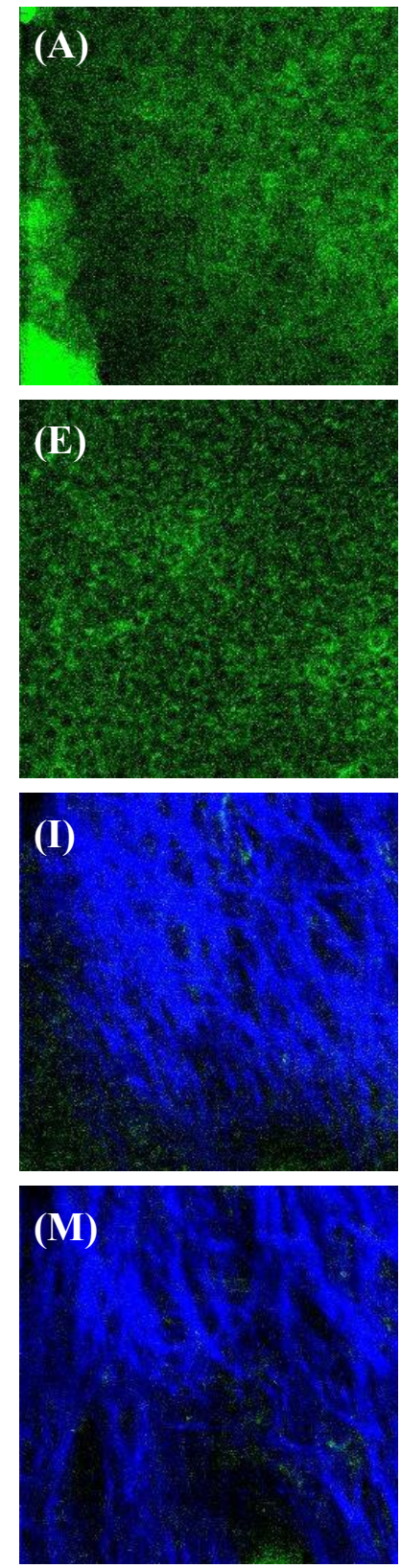
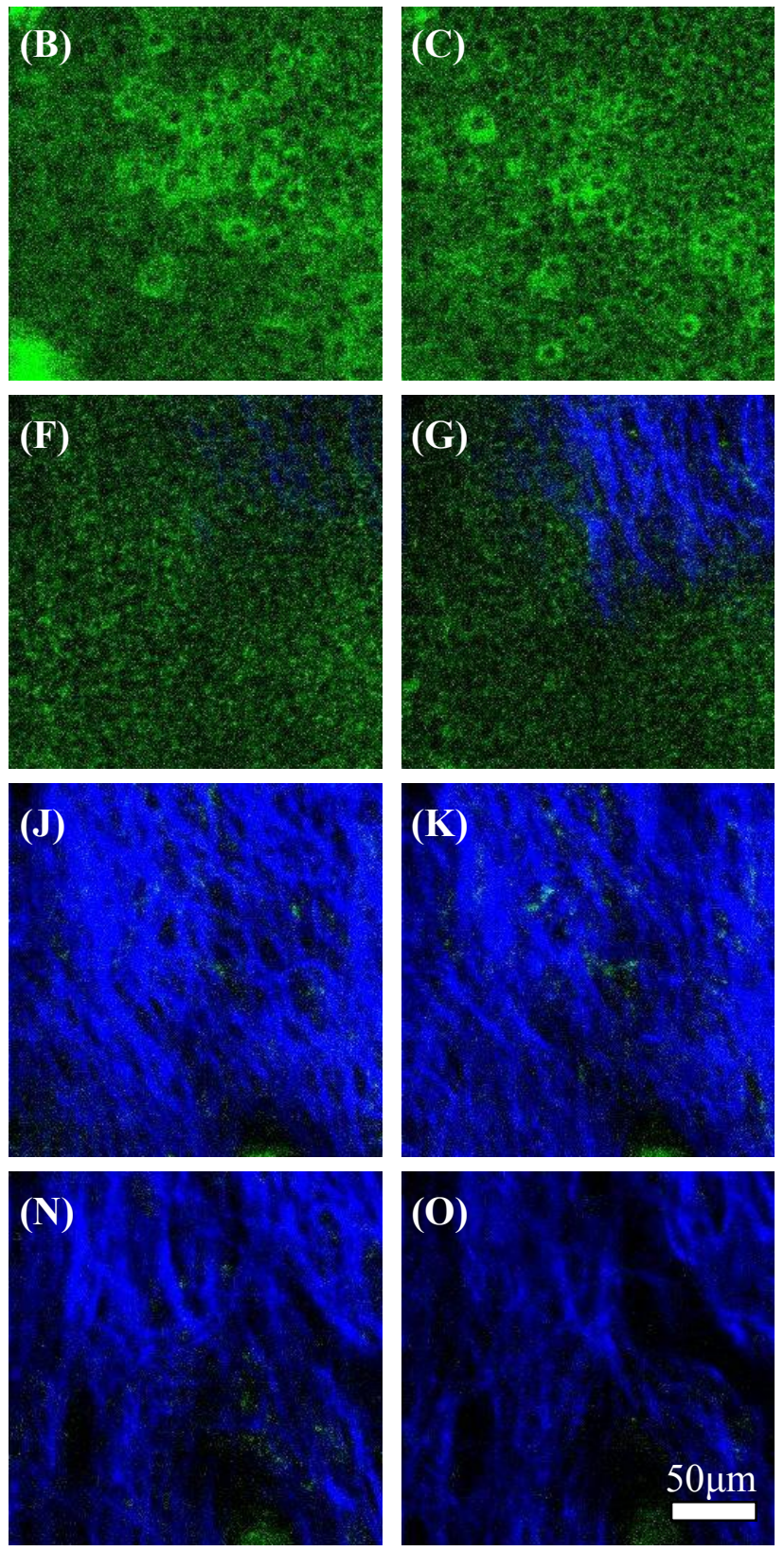

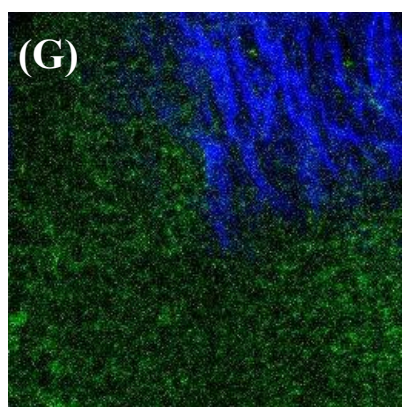

(K)
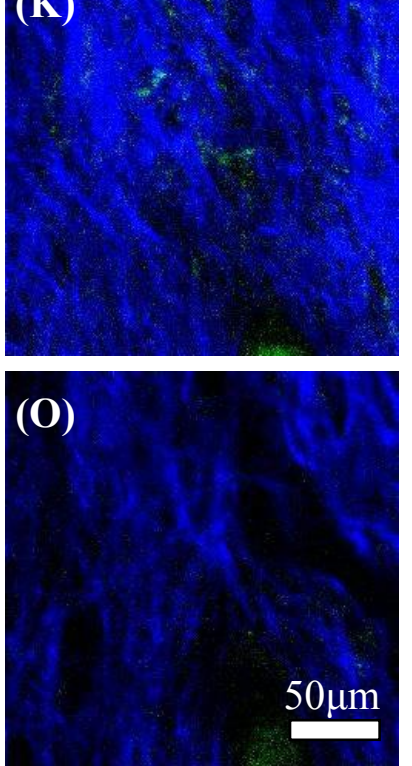

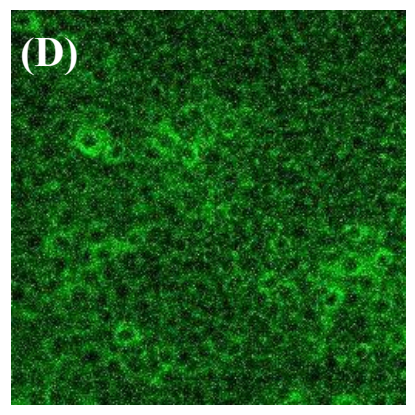

(H)
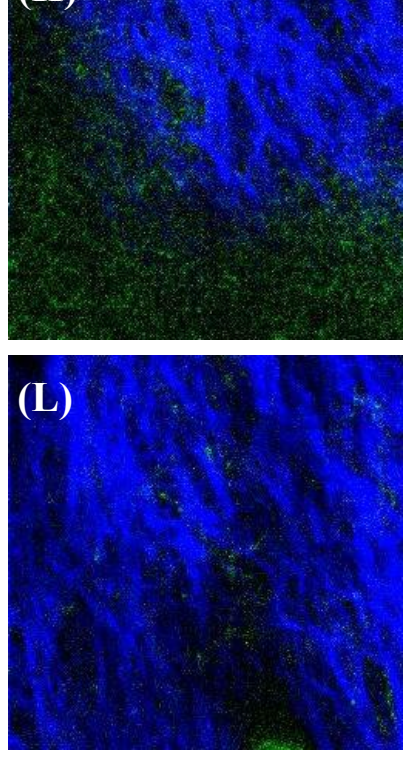

Fig.4. Multiple images of normal nude mice skin obtained at increasing depth of $10 \mu \mathrm{m}$. Green and blue pseudo colors represent two-photon fluorescence and SHG signals respectively.

Compared with normal skin shown in Fig.4, we found that the distribution of squamous cells in DMBA treated skin appear with greater disorder. Furthermore, the images near the surface show that both the shape and size of individual cells are more irregular than those from the normal nude mouse. Finally, the depth when the cells disappear occurs deeper into the dermis in the DMBA treated skin. These characteristics implied that the treated squamous cells are quite abnormal. 

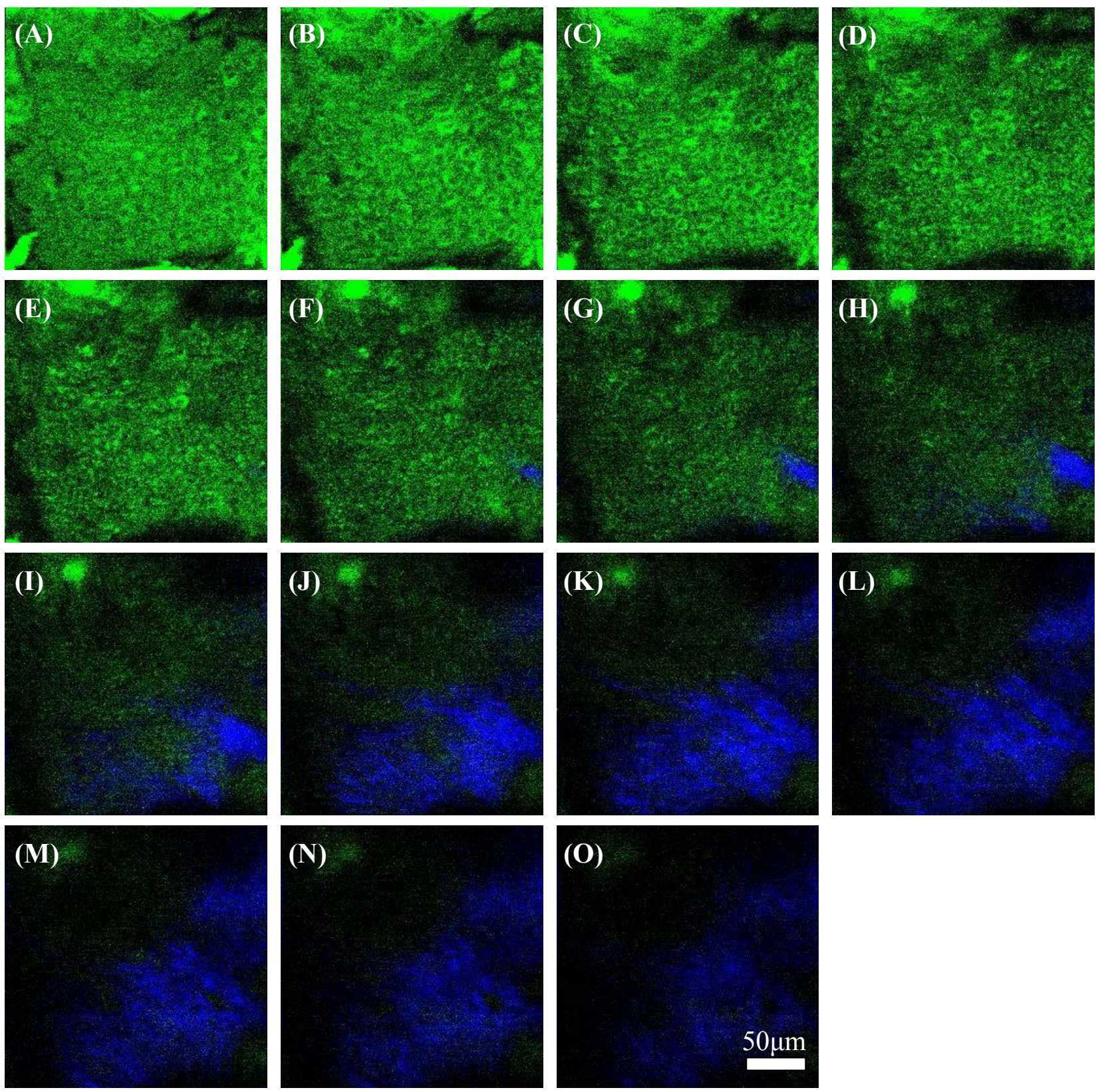

Fig.5. 3D imaging of DMBA treated mice skin and each image interval represents $10 \mu \mathrm{m}$. Green and blue pseudo colors represent two-photon fluorescence and SHG signals respectively.

Another feature is the structural change in the collagen fibrils as revealed by SHG imaging. Network-like arrangement of collagen fibers plays a significant role in connective tissue such as dermis, however, we find that the identification of these connective tissues are more difficult in the DMBA treated skin in comparison to normal dermis.

In order to better delineate DMBA treated skin from normal condition, a quantitative Autofluorescence versus SHG Index (ASI) is developed. The ASI is defined as 


$$
A S I \equiv \frac{A F-S H G}{A F+S H G}
$$

ASI is a competitive value of counted pixels between AF (autofluorescence) and SHG. According to this definition, the ASI approaches the maximum value of 1 when only AF signals are present, such as in the stratum corneum layer. The smallest value of ASI is -1 , and this occurs when AF is absent and only the SHG signal is present, such as in the dermis layer. The index of each 3D image is computed and the average index in each case is calculated.
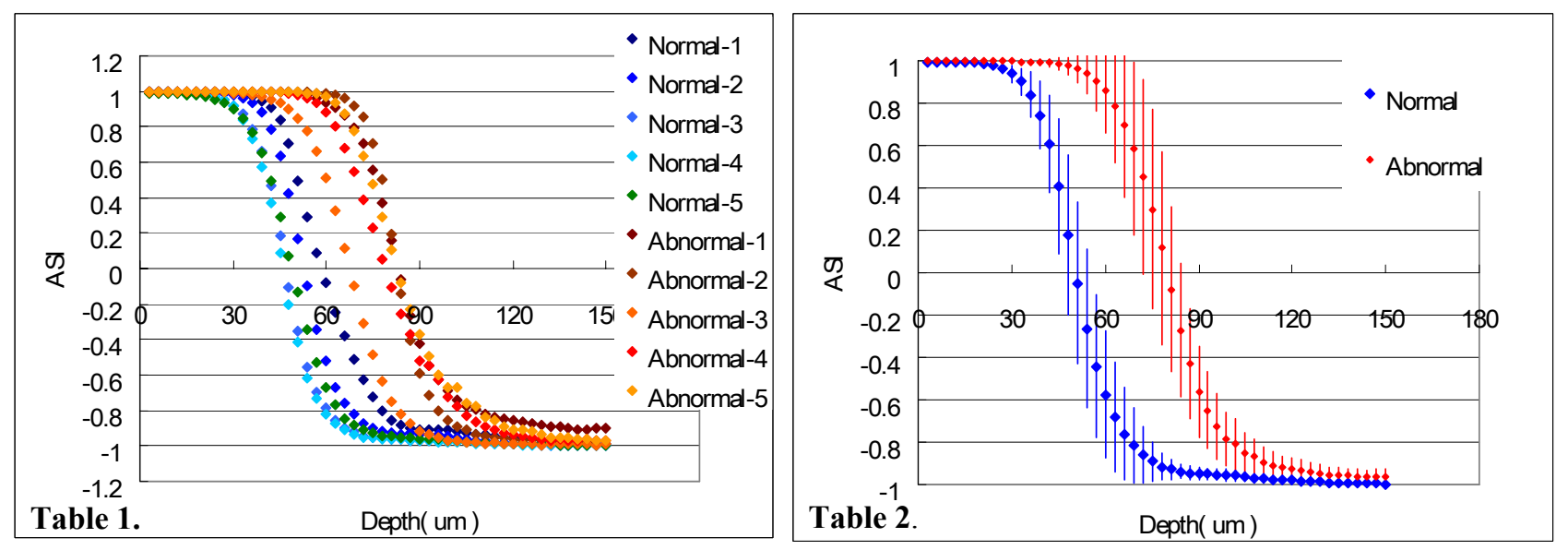

Table 1 and 2 shows the calculated ASI value as a function of depth, for the normal and DMBA treated skin. We found that ASI starts to decline at the depth of $30 \mu \mathrm{m}$ in normal skin. Whereas the ASI for DMBA treated skin does not change until $60 \mu \mathrm{m}$. This can be attributed to the overgrowth of squamous cells due to the application of carcinogen DMBA.

The co-exist phase of AF and SHG is also different in normal and DMBA treated skin. The region where the value of ASI varies from 0.95 to -0.95 is larger in the carcinogen treated skin, and it is consistent with the observation of 3D imaging in vivo.

For comparison, the sections of each specimen were further processed for histological examination with hematoxylin and eosin (H\&E) stains. Fig. 6 shows the H\&E stain of normal skin with similar size of each squamous cell and fibrous collagen network. However, the hyperplasia of squamous cell epithelium can be observed in the DMBA treated skin. The shape and size of squamous cells are not only inhomogeneous but also appear disordered, as shown in Fig. 7. After five weeks of DMBA treatment, the specimen developed squamous precancer tissue. Finally, Fig. 8 shows that carcinogen treated skin had already developed to malignant squamous cell carcinoma (SCC) after seven weeks. ${ }^{5}$
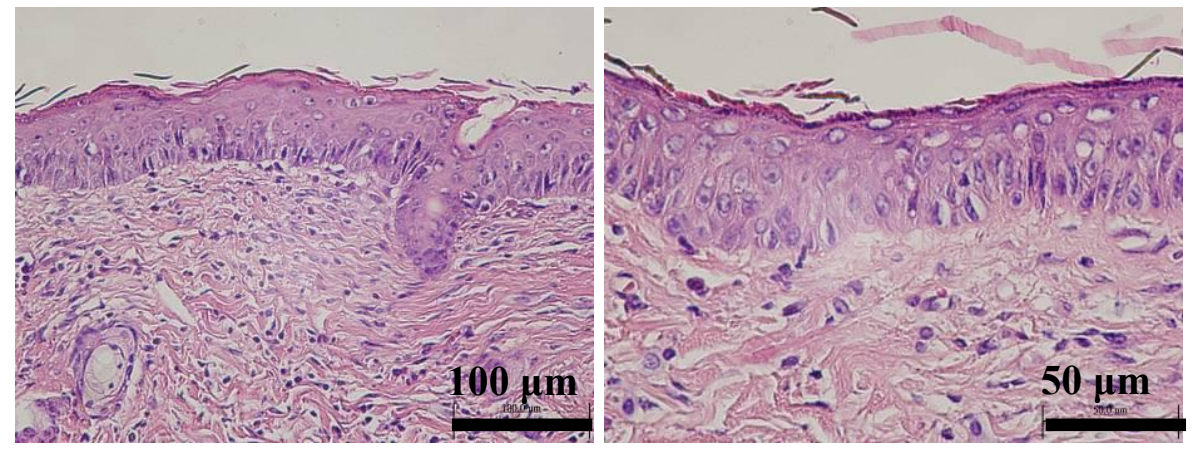

Fig.6. Normal skin of 13-week-old nude mice. 


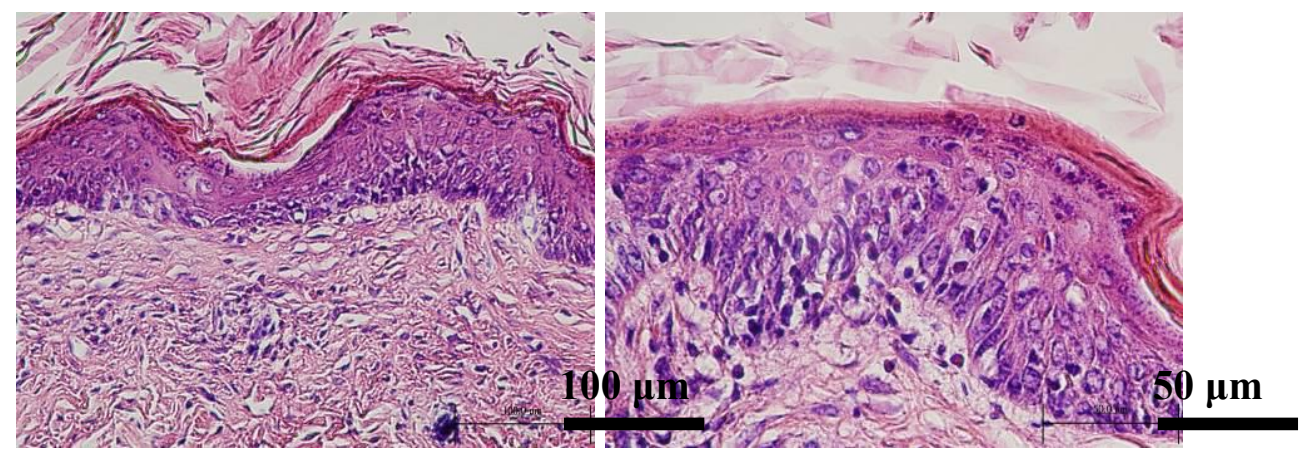

Fig.7. DMBA treated skin of 13-week-old nude mice.

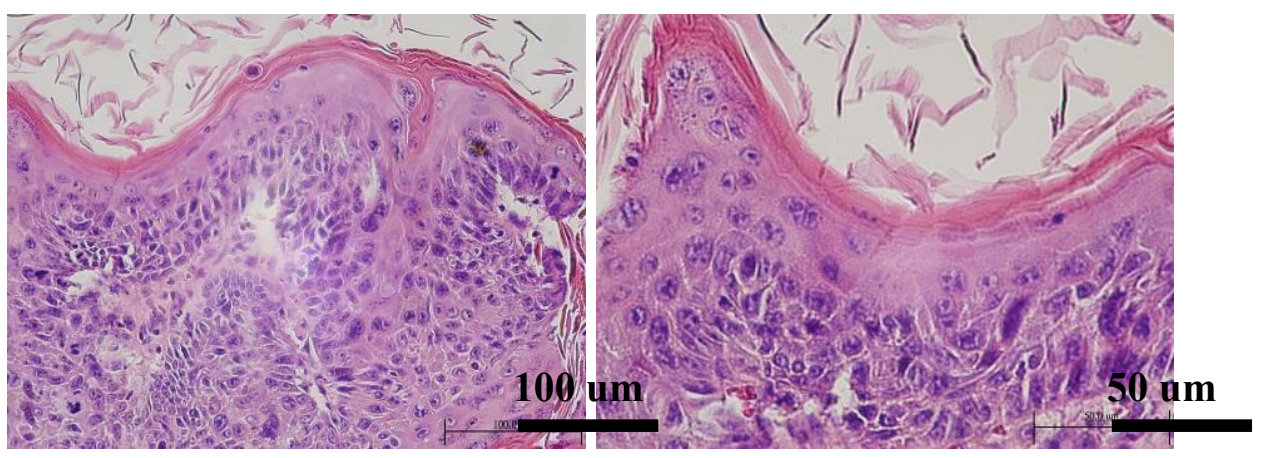

Fig.8. DMBA treated skin of 15-week-old nude mice.

The 3D images are consistent with the histological results. In the DMBA treated tissue, the SHG signal in the dermis is greatly diminished in comparison with that of normal stroma. Since the SHG signal of collagen depends on the regular packing of triple-helical collagen molecules, the diminished SHG signal in the morbid tissue indicated that the collagen structures are disrupted or the collagen molecules are deficient.

It has also been shown that matrix metalloproteinase expression is upregulated in the cancer process, and the collagenolytic activity may lead to the disruption of collagen in the adjacent stroma. ${ }^{6}$

Our result is consistent with a previous report that demonstrated ASI index can be used to differentiate cancer cells and adjacent cancer stroma from the normal dermis. ${ }^{7}$

\section{CONCLUSION}

We have developed a chamber composed of medical-grade titanium alloy for investigating the early development of cancer. Our design allows long-term and large-area in vivo observation in nude mice. Combined with two-photon fluorescence and SHG microscopy, it is possible to obtain three dimensional and high-resolution images with reduced photo-damage and enhanced penetration depth.

With 3D imaging of tissue morphology, we can visualize the interaction between squamous cells and connective tissue such as collagen. By the application of ASI, we can obtain qualitative and quantitative information for distinguishing morbid tissue from normal skin. Our results suggest that two-photon fluorescence and SHG microscopy can be further developed into a noninvasive imaging tool for the clinical evaluation of precancer development. 


\section{ACKNOWLEDGEMENT}

This work was supported by grants from the National Science Council and was completed in the Optical Molecular Imaging Microscopy Core Facility (A5) of NRPGM

\section{REFERENCES}

1. W. Denk, J.H. Strickler, and W.W. Webb, "Two-photon laser scanning fluorescence microscopy," Science 248, 73$76(1990)$.

2. E.B. Brown, R.B. Campbell, Y. Tsuzuki, et al., "In vivo measurement of gene expression, angiogenesis and physiological function in tumors using multiphoton laser scanning microscopy," Nature Medicine 7, 864-868 (2001).

3. F.C. Li, C.C. Wang, S.J. Lin, S.H. Jee, W. Lo, and C.Y. Dong, "Dorsal skin fold chamber for high resolution multiphoton imaging," Optical and quantum electronics 37(13-15), 1439-1445 (2005).

4. L.H. Kligman, and R. Elenitsas, "Melanoma induction in a hairless mouse with short-term application of dimethylbenz[a]anthracene," Melanoma Research 11(4), 319-324 (2001).

5. Edited by C.D.M. Fletcher, Diagnostic histopathology of tumors, Churchill Livingstone, London, 2002.

6. M. Seiki, " Membrane-type 1 matrix metalloproteinase : a key enzyme for tumor invasion," Cancer Letters 194, 111 (2003).

7. S.J. Lin, S.H. Jee, C.J. Kuo, R.J. Wu, et al., " Discrimination of basal cell carcinoma from normal dermal stroma by quantitative multiphoton imaging, " Optics Letters 31(18), 2756-2758 (2006). 